\title{
Evaluation of Brønsted and Lewis acid sites in H-ZSM-5 and H-USY with or without metal modification using probe molecule-synchrotron X-ray powder diffraction
}

\author{
Benedict T.W. Lo, ${ }^{a, b}$ Wei-che Lin, ${ }^{a}$ Molly Meng Jung Li, ${ }^{a, b}$ Sarah Day, ${ }^{c}$ Chiu Tang, ${ }^{c}$ Shik Chi \\ Edman Tsang ${ }^{a^{*}}$ \\ a Wolfson Catalysis Centre, University of Oxford, Oxford, OX1 3QR, UK \\ ${ }^{b}$ Department of Applied Biology and Chemical Technology, Hong Kong Polytechnic \\ University, Hong Kong, China \\ c Diamond Light Source Ltd, Harwell Science and Innovation, Didcot, OX11 ODE, UK
}

\begin{abstract}
The adsorbate interactions of furans as probe molecules in H-ZSM-5 and H-USY with and without metal ion modification have been revealed by synchrotron X-ray powder diffraction (SXRD). The Rietveld refinements give both qualitatively and quantitatively the spatial arrangements and adsorption geometries of furan and 2,5-dimethylfuran (DMF) on the Brønsted acid sites (BAS) and metal Lewis acid sites (LAS). The bonding information between the adsorbate-framework can also be correlated with thermogravimetric findings. The use of probe molecule-SXRD can be used as a new characterisation tool which can spatially interrogate the acidity of crystalline porous catalysts, leading to molecular engineering of new catalytic systems.
\end{abstract}

\section{Key words}

Methanol conversion, SAPO-34 zeolite, synchrotron X-ray powder diffraction, product distribution, surface methoxy surface 


\section{Introduction}

Many industrial processes rely on solid-state heterogeneous catalysts thanks to the ease of product/catalyst separation. Some important catalysts such as iron-based catalysts in the Haber-Bosch process for ammonia synthesis [1]), zeolite-based catalysts for petrochemical refining [2], as well as for conversion of biomass-derived chemicals $[3,4]$ ) have been developed. To guide the design of more superior catalytic materials, understanding surface chemistry especially the internal surface is critical. The surface and catalytic chemistries are however far from simple and have not yet been extensively studied, not to mention the surface molecular structure-activity relationship at an atomistic level.

In crystalline porous catalytic materials such as zeolites, it is challenging to use the traditional characterisation techniques to probe the internal surface. Silicate in origin, zeolites can be partially substituted with $\mathrm{Al}$, forming a microporous framework made of $\mathrm{TO}_{4}$ tetrahedra. Brønsted acid sites (BASs) are hence generated on the internal zeolite framework to compensate the charge imbalance [5]. The surface chemistry on the inner porous walls of zeolites can, therefore, be understood as acid-base interactions between the basic adsorbate species and acidic framework BASs leading to their protonation[6]. This interaction can be referred to as the adsorption strength and geometry of adsorbate in the zeolite. (i) The former is merely based on how tightly the adsorbate and framework BASs are bound together. Combined thermal (such as temperature-programmed desorption (TPD), thermogravimetric analysis (TGA)) and spectroscopic techniques (such as infra-red (IR) and nuclear magnetic resonance (NMR) spectroscopy) can extensively characterise the strength of the zeolite sorption chemistry[7]. (ii) Whereas, the latter can be affected by the intrinsic steric properties of both the adsorbate molecule and the nature of the adsorption sites. The spatial properties of a BAS on the internal surface of zeolites and a metal Lewis acid site (LAS) that is coordinated with the adsorbate are different.

The conversion of biomass-derived molecules to useful chemicals has gained increasing attention, such as the zeolite-catalysed conversion of furans to platform aromatic compounds [8]. The improvement in characterisation techniques has allowed much more rational approaches in new catalyst design $[3,4,9]$. Recently, the adsorbate-structure interactions in microporous catalysts have been studied using on synchrotron X-ray powder diffraction (SXRD) and Rietveld refinement in combination with theoretical calculations [10-13]. Even 
biomass-based adsorbate molecules can be detected at an atomistic resolution within experimental error, which is caused by the slight but significant alteration in scattering parameters upon adsorption on the repeatable internal porous crystalline surface [13-16]. It is also found that both the intrinsic Brønsted acidity and the geometry for adsorption of the probe molecules with respect to the framework structure can offer an understanding of molecular interactions of some important zeolite-catalysed processes $[17,18]$.

Herein, by combining TGA with the Rietveld refinements of SXRD, this work has investigated the adsorbate-framework interactions of furan and 2,5-dimethylfuran as probe molecules in H-ZSM-5 and H-USY at atomistic resolution. A clear and rational spatial relationship between the adsorbates and BAS/LAS in H-USY and H-ZSM-5 zeolites is established. As a result, by using a suitable model molecule to mimic reactant molecule(s) on zeolite-based catalysts, the structure-activity relationship and mechanistic insights can be inferred. 


\section{Results and discussion}

High-quality H-USY and H-ZSM-5 samples are commercially available. The structural properties of H-USY (FAU, Si/AI = 7.3, from TOSOH) and H-ZSM-5 (MFI, Si/AI = 19.3, from SRIPT-SINOPEC) zeolites and their respective structural properties were previously reported (see Figure S1 in the Supporting Information for the microscopy images)[3]. Through ionexchange of $\mathrm{Ag}^{+}$and $\mathrm{Zn}^{2+}$ with H-ZSM-5, Ag-ZSM-5 and Zn-ZSM-5 were prepared. To reduce migration of the metal sites, the samples were not calcined. The content of $\mathrm{Ag}, \mathrm{Cu}$, and $\mathrm{Zn}$ atom per unit cell of H-ZSM-5 are 1.67(3), 1.89(3) and 1.97(4), respectively (as determined by the Rietveld refinement of the high-resolution SXRD data, which will be discussed later). The extents of metal incorporation are comparable to each other.

The adsorption strengths between the zeolites with furan $\left(\mathrm{C}_{4} \mathrm{H}_{4} \mathrm{O}\right)$ and 2,5-dimethylfuran (DMF, $\mathrm{C}_{6} \mathrm{H}_{8} \mathrm{O}$ ) were revealed by TGA (Figure S2). Furan and DMF were pre-adsorbed on $\mathrm{H}-\mathrm{USY}$, H-ZSM-5, Ag-ZSM-5 and Zn-ZSM-5 at $25^{\circ} \mathrm{C}$. The broad desorption peaks in TGA are commonly observed due to the diffusion of desorbed substrate molecules within the microporous nature of the zeolitic frameworks. The basicity of these probes in terms of proton affinity and gasphase basicity from various literature are presented in Table 1. DMF is inherently much more basic than furan due to the induction effect from the two extra substituent methyl groups[19]. The peak desorption temperatures are summarised in Table 1, whereas the TGA profiles are summarised in Figure S3. It can be seen that DMF desorbs from zeolites at higher temperature by $c a .40^{\circ} \mathrm{C}$ than furan in all the samples. Upon the desorption of DMF from the samples, the $H(B A S) \cdots D M F$ interaction will be broken from the $\mathrm{O}_{\text {framework }}-\mathrm{H}$-..DMF adduct as similarly to $\mathrm{H} \cdots$ furan from $\mathrm{O}_{\text {framework }}-\mathrm{H} \cdots$ furan. This agrees with our expectation that more thermal energy is required to break the stronger acid-base interactions. As DMF is more basic than furan, it therefore desorbs at a higher temperature. A similar TGA profile was reported from our desorption study of pyridine from H-ZSM-5 [17]. Thus, the desorption behaviour, as determined by TGA, also agrees with the previous study of pyridine on H-ZSM-5.

From the levelling of the thermogravimetric baseline, the content of chemisorbed furan and DMF desorbed from the samples were summarised in Table 2. The content desorbed from $\mathrm{H}-$ ZSM-5 was measured as ca. $69 \mathrm{mg} \mathrm{gH}_{\mathrm{H}-\mathrm{ZSM}-5^{-1}}\left(0.91 \mathrm{mmol}_{\left.\mathrm{H}-\mathrm{ZSM}-5^{-1}\right)}\right.$ and $82 \mathrm{mg}{\mathrm{gH}-\mathrm{ZSM}-5^{-1}}(0.86$ mmol $g_{H-Z S M-5}{ }^{-1}$ ) from 250 to $600{ }^{\circ} \mathrm{C}$. This corresponds to 1.01 furan and 0.95 DMF molecules per BAS. It infers to the stoichiometric strong acid-base interactions between furans and the 
protonic BASs. As seen in the quantity of adsorbed furan and DMF in H-USY, Ag- and Zn-ZSM5, although a slightly larger discrepancy is shown in the Zn-ZSM-5 seen, comparable quantities of adsorbed furan and DMF were measured in $\mathrm{H}$ - and Ag-ZSM-5 based on the thermogravimetric analysis (TGA) results within experimental errors. This suggests that the adsorption behaviours of the Lewis basic probe molecules on the acidic sites are similar in nature. On the other hand, the quantity of BASs of the H-USY was determined as $0.1 \mathrm{mmol} \mathrm{\textrm {g } ^ { - }}$ 1; the notable difference between the quantity of the BASs and adsorbates (furan: $0.82 \mathrm{mmol}$ $\mathrm{g}^{-1}$ and DMF: $1.49 \mathrm{mmol} \mathrm{g}^{-1}$ ) can be indicative of the formation of organic cluster structures within the spacious void of H-USY. We ascribed to the formation of cluster structures in the void space in H-USY due to the voids created in this structure by the heat-treatment in steam. More organic like DMF can form extensive cluster structures in the gaseous and liquid phases based on computation calculations[20,21], where furan molecules are linked together via $\mathrm{H}$ bonding interactions. The internal void space of $\mathrm{H}-\mathrm{USY}$ is much larger than that in $\mathrm{H}-\mathrm{ZSM}-5$ (cf. 11.9 vs $7.0 \AA$ A)[22], which can offer a much greater spatial freedom to facilitate the formation of furan clusters. It infers that a large proportion of the furans is located closer to the central space of the spherical void. The difference in adsorption quantity between furan and DMF greatly exceeds typical experimental errors, $c f$. adsorbed DMF is 1.82 times greater than adsorbed furan (verified by our repeated experiments). It can be attributed to the ability in forming cluster of furans with different electron densities. Due to the additional presence of the two methyl substituent groups, the aromatic furan ring is more electron rich, which can promote further interactions.

Meanwhile, an increase in the peak desorption temperature can be observed when $\mathrm{Ag}^{+}$and $\mathrm{Zn}^{2+}$ ions were ion-exchanged with the BAS of the H-ZSM-5 zeolite by $c a .25$ and $75{ }^{\circ} \mathrm{C}$ respectively. The peak desorption temperatures of furans from Ag-ZSM-5 and Zn-ZSM-5 are notably higher than those from H-ZSM-5. It can be ascribed to the stronger binding interactions between furans with $\mathrm{Ag}^{+}$and $\mathrm{Zn}^{2+}$ than with the BASs, presumably due to greater effective nuclear charge $\left(Z_{\text {eff }}\right)$ of the cations and the degree of covalency. The strong interaction between metal LAS and Lewis basic furan and DMF could also be understood as simply adsorption or ligand substitution (displacement of water by furan or DMF). Notice that, even though the strength of the acid-base interaction in zeolites can be gravimetrically 
probed by TGA, the spatial information about the adsorbate species with respect to the zeolite framework remains elusive.

Table 1. Basicity values from literature, and the peak desorption temperatures of DMF and furan from H-USY, H-ZSM-5, Ag-ZSM-5 and Zn-ZSM-5 zeolites from the differential of the TGA results.

\begin{tabular}{|c|c|c|c|c|c|c|}
\hline & & & \multicolumn{4}{|c|}{ Peak desorption temperature $\left({ }^{\circ} \mathrm{C}\right)$} \\
\hline $\begin{array}{l}\text { Adsorbate } \\
\text { species }\end{array}$ & $\begin{array}{l}\text { Proton Affinity } \\
\left(\mathrm{kJ} \mathrm{mol}^{-1}\right)[23]\end{array}$ & $\begin{array}{l}\text { Gas-Phase } \\
\text { Basicity } \\
\left(\mathrm{kJ} \mathrm{mol}^{-1}\right) \text { [23] }\end{array}$ & H-USY & $\begin{array}{l}\text { H-ZSM- } \\
5\end{array}$ & $\begin{array}{l}\mathrm{Ag}- \\
\mathrm{ZSM}-5\end{array}$ & $\begin{array}{l}\text { Zn- } \\
\text { ZSM-5 }\end{array}$ \\
\hline DMF & 835.2 & 865.9 & 340 & 355 & 381 & 437 \\
\hline Furan & 770.9 & 803.4 & 298 & 310 & 337 & 384 \\
\hline
\end{tabular}

Table 2. The content of adsorbed furan and DMF in the zeolite samples.

\begin{tabular}{|l|l|l|l|l|}
\hline Sample & $\begin{array}{l}\text { Furan }(\mathrm{mg} \\
\left.\mathrm{g}_{\text {sample }}{ }^{-1}\right)\end{array}$ & $\begin{array}{l}\text { DMF (mg gample } \\
1)\end{array}$ & $\begin{array}{l}\text { Furan (mmol } \\
\left.\mathrm{g}_{\text {sample }}{ }^{-1}\right)\end{array}$ & $\begin{array}{l}\text { DMF (mmol } \\
\mathrm{g}_{\text {sample }}{ }^{-1}\end{array}$ \\
\hline H-USY & 56 & 143 & 0.82 & 1.49 \\
\hline H-ZSM-5 & 69 & 82 & 0.91 & 0.86 \\
\hline Ag-ZSM-5 & 58 & 73 & 0.85 & 0.76 \\
\hline Zn-ZSM-5 & 46 & 84 & 0.67 & 0.87 \\
\hline
\end{tabular}

Noticeably, the Rietveld refinement of the SXRD patterns could enable the direct visualisation of the adsorbate structures with respect to the zeolitic framework. The synchrotron data were collected on Beamline I11 at Diamond Light Source (UK). The X-ray energy of the incident beam was tuned to $15 \mathrm{keV}$ with the calibrated 0.825251 (2) $\AA$ (Beamline I11) [24]. The tuned energy for each beamline emits the optimum X-ray flux to achieve high contrast (signal-tonoise ratio) as well as high angular resolution. The SXRD patterns and the Rietveld refinements of furan and DMF pre-adsorbed on $\mathrm{H}-\mathrm{USY}$ and $\mathrm{H}-\mathrm{ZSM}-5$ are presented in Figure 1. The crystallographic parameters are presented in Table S3. Note that the zeolites were pretreated at $200^{\circ} \mathrm{C}$ to remove most physisorbed adsorbates. Briefly, the adsorption of furan and DMF on $\mathrm{H}-\mathrm{ZSM}-5$ causes slight unit cell contraction by $c a .0 .3 \%$ and $0.2 \%$ respectively. In contrast, the adsorption of furan and DMF on H-USY expands the H-USY lattice by $0.07 \%$ and $0.09 \%$ respectively. It could be due to the much larger porous aperture of H-USY (c.f. $1.3 \mathrm{~nm}$ 
vs $0.8 \mathrm{~nm}$ of $\mathrm{H}-\mathrm{ZSM}-5$ ), leading to the much less affected local host structure upon furans adsorption.

The Rietveld refinements of SXRD data reveals the furan adsorbates with respect to the crystal host-structure. The Fourier contrast map of DMF pre-adsorbed in H-ZSM-5 is presented in Figure 2. As $4190 \mathrm{hkl}$ reflections were used for the refinement calculations of the H-ZSM-5 samples (870 hkl for $\mathrm{H}$-USY), ca. 150 atomic and structural parameters (44 parameters for $\mathrm{H}$ USY) could therefore be refined in a statistic reliable manner. We note the higher uncertainty in terms of the atomic parameters of the adsorbate species than the rigid framework $\mathrm{T}$ and $\mathrm{O}$ atoms due to their inherently higher degrees of freedom of the adsorbates than that of the framework atoms. To best simulate furan DMF for Rietveld refinement, rigid body Z-matrices were used with the isotropic displacement factors $\left(B_{\text {eq }}\right)$ fixed at $8 \AA^{2}$ arbitrarily to represent the averaging of the adsorbate positions (see details in the experimental section and the Rietveld refinement verification in Tables S3-S4) $[3,4,25,26]$. Supported by the good refinement fit (the small discrepancies in the grey difference profiles) and indicated by lowreliability R-factors $\left(R_{w p}\right)$, the detailed structural parameters are presented in Table S3. Similar to our previous work that studies DMF on $\mathrm{H}-\mathrm{ZSM}-5$, furan on $\mathrm{H}$-USY, as well as $\mathrm{Y}$-valerolactone on $\mathrm{Zn}-\mathrm{ZSM}-5$, the rigid framework $\mathrm{O}$ and metal centres are thus chosen as the crystallographic pointers to measure the interatomic distances between the adsorption species and the framework $\mathrm{O}$ atoms.
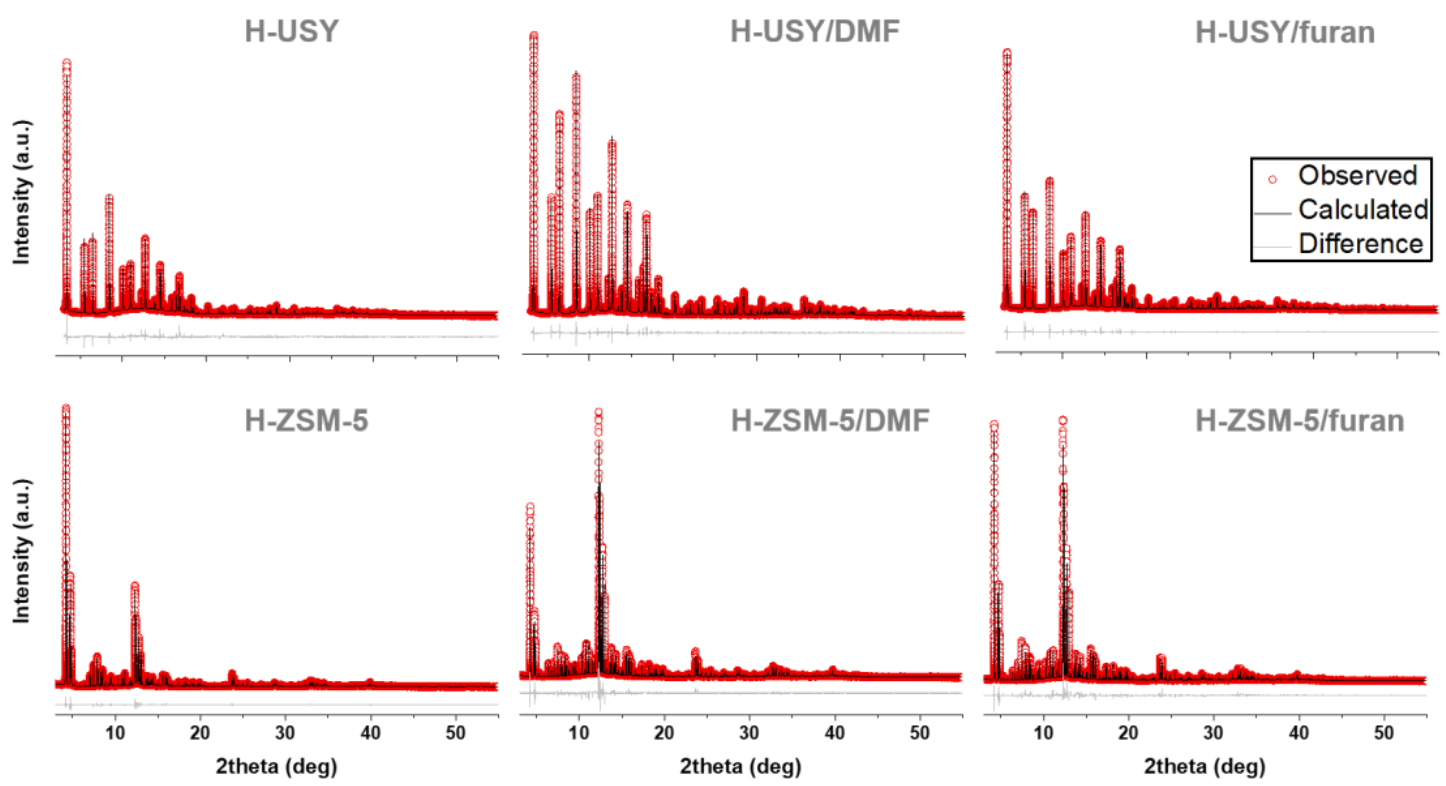
Figure 1. The SXRD patterns and Rietveld refinement profiles of pristine, DMF and furan preadsorbed on $\mathrm{H}-\mathrm{ZSM}-5$ and $\mathrm{H}$-USY at $25^{\circ} \mathrm{C}$ (pre-treated at $200{ }^{\circ} \mathrm{C}$ to remove physisorbed adsorbates). The SXRD patterns were measured at Diamond Light Source Beamline I11 using the multi-analyser crystals detector. The crystallographic are summarised in Table S3.

For a better illustration, the Rietveld refined crystal structures are presented in Figure 3 . The detailed atomic parameters are presented in Table S5. As determined in our previous work, the BAS (hydroxyl) and Al of this H-ZSM-5 were found at the framework 018 and T6 crystallographic positions. For the $\mathrm{H}$-USY, the BAS was found at the framework $\mathrm{O} 4$ crystallographic position. The Rietveld refinements of the SXRD data collected on DMF and furan pre-adsorbed on H-USY revealed an independent binding site at the Wyckoff position of $96 \mathrm{~h}$. Whereas, for H-ZSM-5, two DMF and furan adsorption sites were determined at the Wyckoff position of $4 c$, with Site 1 located at the straight-sinusoidal cross-channel region and Site 2 at the straight channel region of the framework. For DMF pre-adsorbed on H-USY (denoted as 'H-USY/DMF'), the closest interatomic distance was measured between $\mathrm{O}_{\mathrm{DMF}}$ and the framework 04 site, as $04-\mathrm{O}_{\mathrm{DMF}}=2.94(2) \AA$. It is notably shorter than that measured in $\mathrm{H}$ USY/furan with O4-O furan $=3.53(2) \AA$. The same trend was also measured in H-ZSM-5/DMF and $\mathrm{H}-\mathrm{ZSM}-5 /$ furan, with $018-\mathrm{O}_{\mathrm{DMF}}=2.57(2) \AA$ and $018-\mathrm{O}_{\text {furan }}=2.74(2) \AA$. Similar to our previous work, Site 2 DMF and furan are located deep in the straight channel in H-ZSM-5, with the refined interatomic distances $c a .3 .8 \AA[3,4]$. The quantities of the adsorbate furans refined from the Rietveld refinements were determined as ca. 1 per BAS, which agrees with the values from the TGA measurements. The shorter refined $\mathrm{O}_{D M F}-\mathrm{O}_{\text {framework }}$ interatomic distances in both H-USY and H-ZSM-5 suggest stronger adsorption interactions.

The effect of zeolite framework type was further been studied. The interatomic distances $O_{\text {framework- }}(\mathrm{H}(\delta+)) \ldots \mathrm{O}_{\text {adsorbate }}\left(\delta\right.$-) in H-ZSM-5/DMF and H-ZSM-5/furan $\left(018-\mathrm{O}_{\mathrm{DMF}}=2.57(2) \AA\right.$ and O18- $\mathrm{O}_{\text {furan }}=2.74(2) \AA$ ) are shorter than those in H-USY/DMF and H-USY/furan, with O4-O $\mathrm{O}_{D M F}$ $=2.94(2) \AA$ and $04-\mathrm{O}_{\text {furan }}=3.53(2) \AA$. The Rietveld refinements are consistent with the understanding that the acid-base interaction can be reflected by the bonding information. It can also be concluded from both TGA and SXRD findings that H-ZSM-5 has more acidic BASs than $\mathrm{H}$-USY. The BAS strengths in different zeolites can be probed by thermogravimetric and spectroscopic techniques, and also by the turnover frequency of n-hexane cracking [27]. More acidic BASs can readily protonate the incoming molecules, hence lowering the activation 
barrier to a greater extent. Based on computation findings, the more acute is the $\angle \mathrm{Al}-\mathrm{O}-\mathrm{Si}$, the more acidic is the protonic BAS [28]. As reported in many literatures the BAS strengths are highly dependent on the framework types. $\mathrm{H}-\mathrm{ZSM}-5$ are more acidic than $\mathrm{H}-\mathrm{USY}$, where the $\angle \mathrm{T}-\mathrm{O}-\mathrm{T}$ in H-ZSM-5 (MFI framework type) are more acute than those in H-USY (FAU framework type) [29].
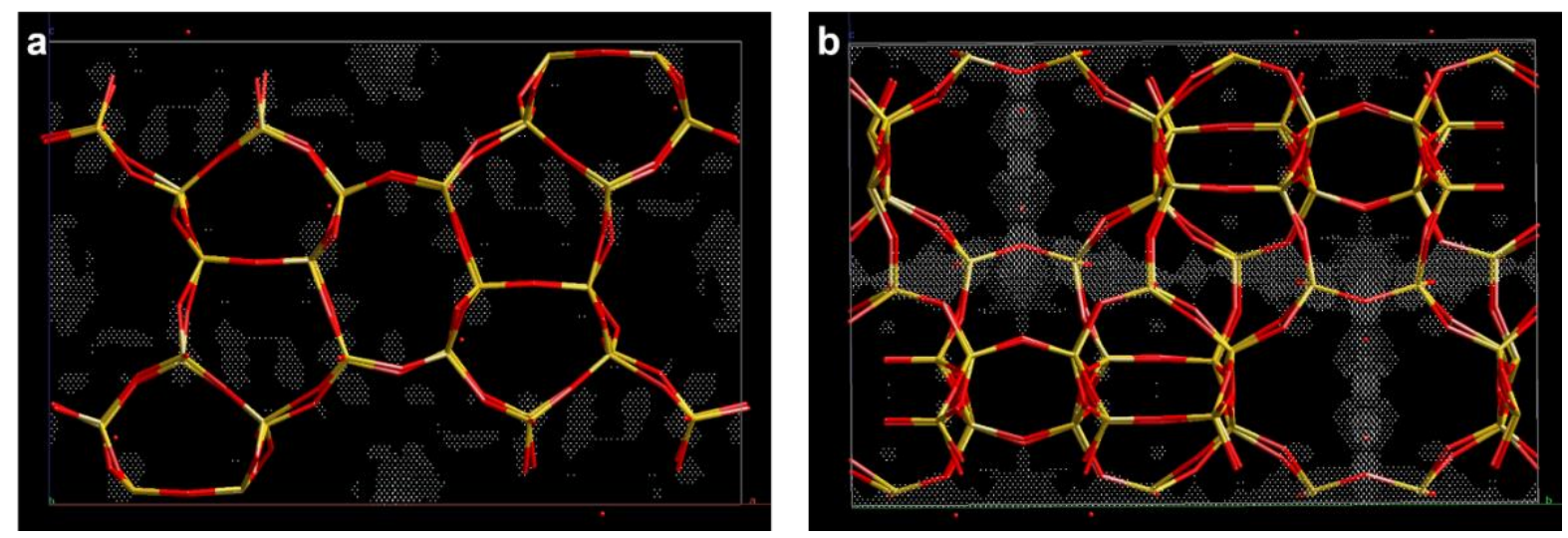

Figure 2. Fourier contrast map of $\mathrm{H}-\mathrm{ZSM}-5$ pre-adsorbed with DMF measured at $25{ }^{\circ} \mathrm{C}$ was generated using the TOPAS software by fixing the framework atoms but without the inclusion of any guest species, viewing from (a) [010] and (b) [100].
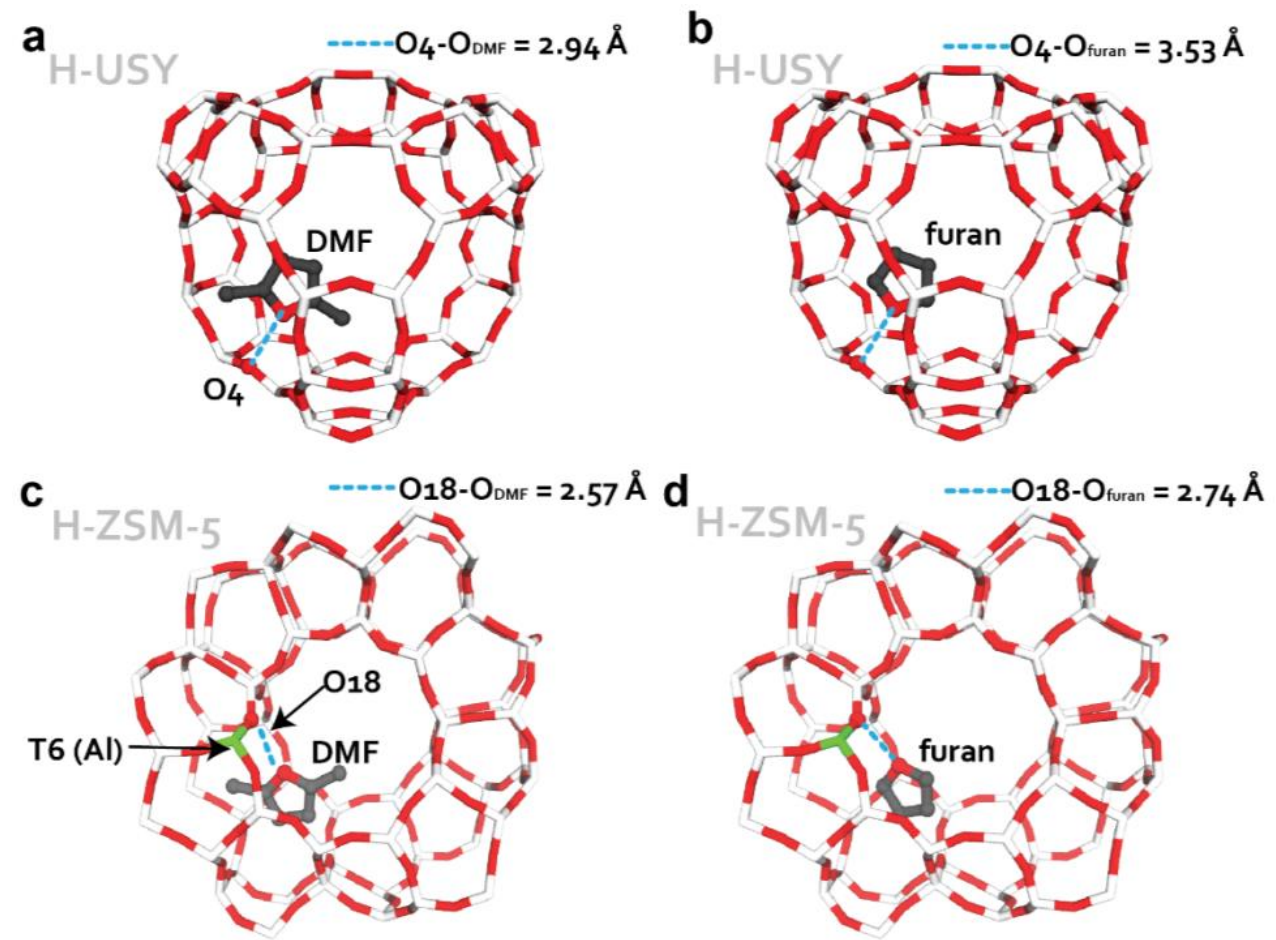

Figure 3. The Rietveld refined crystal structures of (a) H-USY/DMF and (b) H-USY/furan (c, d) (c) H-ZSM-5/DMF and (d) H-ZSM-5/furan. Specific directions (as labelled) are selected to show the super-cage of $\boldsymbol{F A U}$ ([111] direction) and the 10-membered ring window opening of $\boldsymbol{M F I}$ 
([010] direction). For clarity, the symmetry of the extra-framework species is disregarded. Ball-and-stick model: white $=\mathrm{Si}$, red $=\mathrm{O}$, black $=\mathrm{C}$. No hydrogens are plotted for clarity.

The bonding information between transition metal ion LAS with DMF and furan adsorbates was similarly investigated. The SXRD patterns and the Rietveld refinement profiles of the $\mathrm{Ag}^{+} / \mathrm{Zn}^{2+}$-exchanged ZSM-5 pre-adsorbed with DMF and furan are presented in Figure 4. A clear difference in Bragg's peak intensities has allowed reliable refinements for the differentiation of DMF and furan interaction with the $\mathrm{Ag}^{+}$and $\mathrm{Zn}^{2+}$ sites. Despite $\mathrm{Ag}^{+}$-aqua complex typically adopts the linear/tetrahedral geometry [30] and $\mathrm{Zn}^{2+}$-aqua adopts the octahedral coordination [31] but its actual geometry of the metal ion LAS sites may change upon furan/DMF adsorption. For instance, octahedral $\left[\mathrm{Zn}\left(\mathrm{H}_{2} \mathrm{O}\right)_{6}\right]^{2+}$ changes to tetrahedral $\left[\mathrm{ZnCl}_{4}\right]^{2-}$ upon a high concentration of chloride in solution. There are also numerous examples of coordination alteration upon ligand substitution dependent on ligand field effect, electrostatic repulsion and relative size. We have accordingly employed the Rietveld refinement-simulated annealing technique to determine the actual coordination geometry. For example, in the refinement attempts in fitting octahedral complexes (rigid-bodies Zmatrices with furan or DMF occupying one ligand site), proximate R-factors were obtained. By fixing furan/DMF as a ligand (we have found the close stoichiometry) and carefully refining site occupancy factors (SOFs) of the remaining five $\mathrm{O}_{\mathrm{H} 2 \mathrm{O}}$ ligand sites in the octahedral Zn-ZSM5 , the SOFs of two $\mathrm{O}_{\mathrm{H} 2 \mathrm{O}}$ ligand sites tend to approach zero while the other three other $\mathrm{O}_{\mathrm{H} 2 \mathrm{O}}$ ligand sites remain relatively unchanged (see Table S4). In contrast, over the repeated refinements of the SOFs of the three $\mathrm{O}_{\mathrm{H} 2 \mathrm{O}}$ ligand sites with tetrahedral metal coordination, the SOFs remain relatively unchanged. It implies that more likely that the metal species $(\delta+)$ should be in the tetrahedral coordination geometry upon a furan $(\delta$-) adsorption. This agrees well that the substitution of water by the bulkier ligand with different polarity can change the metal ion coordination geometry from octahedral to tetrahedral form. There was no change in the tetrahedral geometry of Ag upon the furan/DMF adsorption on Ag-ZSM-5.

As seen in the Rietveld refined crystal structures presented in Figure 5, there are two metal ion sites in Ag-ZSM-5 and Zn-ZSM-5, with Site 1 (Ag1 and Zn1) in the sinusoidal channel and Site 2 (Ag2 and Zn2) in the straight/sinusoidal cross-channel intersection. For Site 1 (Wyckoff $4 c$ ), the bond distances were determined as: $\mathrm{O}_{\mathrm{DMF} 1} \mathrm{Ag} 1=2.59(3) \AA, \mathrm{O}_{\text {furan1 }} \mathrm{Ag} 1=2.66(2) \AA$, $\mathrm{O}_{\text {DMF1 }}-\mathrm{Zn} 1=2.23(2) \AA$ and $\mathrm{O}_{\text {furan1 } 1} \mathrm{Zn} 1=2.57(2) \AA$; these bond distances are notably longer 
than those at Site 2 (Wyckoff $4 c$, c.f. $\mathrm{O}_{\mathrm{DMF} 2} / \mathrm{O}_{\text {furan2 }} \mathrm{Ag} 2=1.99(4) \AA$ and $2.25(2) \AA$, and $\mathrm{O}_{\mathrm{DMF} 2} / \mathrm{O}_{\text {furan2-Zn2 }}=2.05(2) \AA$ and $2.29(2) \AA$ ). The refined bond distances are in general good agreement with the cation- $\mathrm{O}$ distances from the computational work by Nikbin et al. who mainly studied the proton and sodium cation forms [32] In addition, a cross-comparison with the $\mathrm{M}-\mathrm{N}$ bonds in various the metal-pyridine/imidazole complexes, was extensively discussed by Strub.[33] Interesting, similar trend has also been observed in the H-ZSM-5 and H-USY, where the refined bond distances between the metal complexes pre-adsorbed with DMF are shorter than those pre-adsorbed with furan due to the difference in the probe molecule basicity. Also, the differences in the bond distances and spatial relationships between Site 1 and Site 2 reflect the potential steric effect by the confined zeolitic framework. The sinusoidal channel is more spatially constrained for large molecules, whereas the steric effect is much less substantial in the straight/sinusoidal cross-channel region. It has been found in our previous work concerning pyridine on H-ZSM-5, the pyridine Site 2 (located in the straight/sinusoidal cross-channel region) is the kinetic intermediate site before filling or and emptying of Site 1 in the sinusoidal channel [17]. Therefore, even for metal ion LAS (such as $\mathrm{Ag}^{+}$and $\mathrm{Zn}^{2+}$ ), a similar steric hindrance on the relatively large adsorption molecules (DMF and furan) remains significant. On the contrary, the straight/sinusoidal cross-channel intersection is sufficiently larger (c.f. pore aperture of $c a .5 \AA$ vs $8 \AA$ ) to better accommodate the DMF- and furan-exchanged metal complexes. Interestingly, even with the addition of metal LAS, it is found that the basicity of the furans also plays a critical role. Comparing ODMF1$\mathrm{Zn1}\left(2.23 \AA\right.$ ) with $\mathrm{O}_{\text {furan1-Zn1 }}(2.57 \AA$ ) $), \mathrm{O}_{\text {DMF1 }}-\mathrm{Zn} 1$ is notably shorter than $\mathrm{O}_{\text {furan1 }}-\mathrm{Zn} 1$ by $0.34 \AA$. Similar differences are also observed in all the sites in both Ag- and Zn-ZSM-5. 


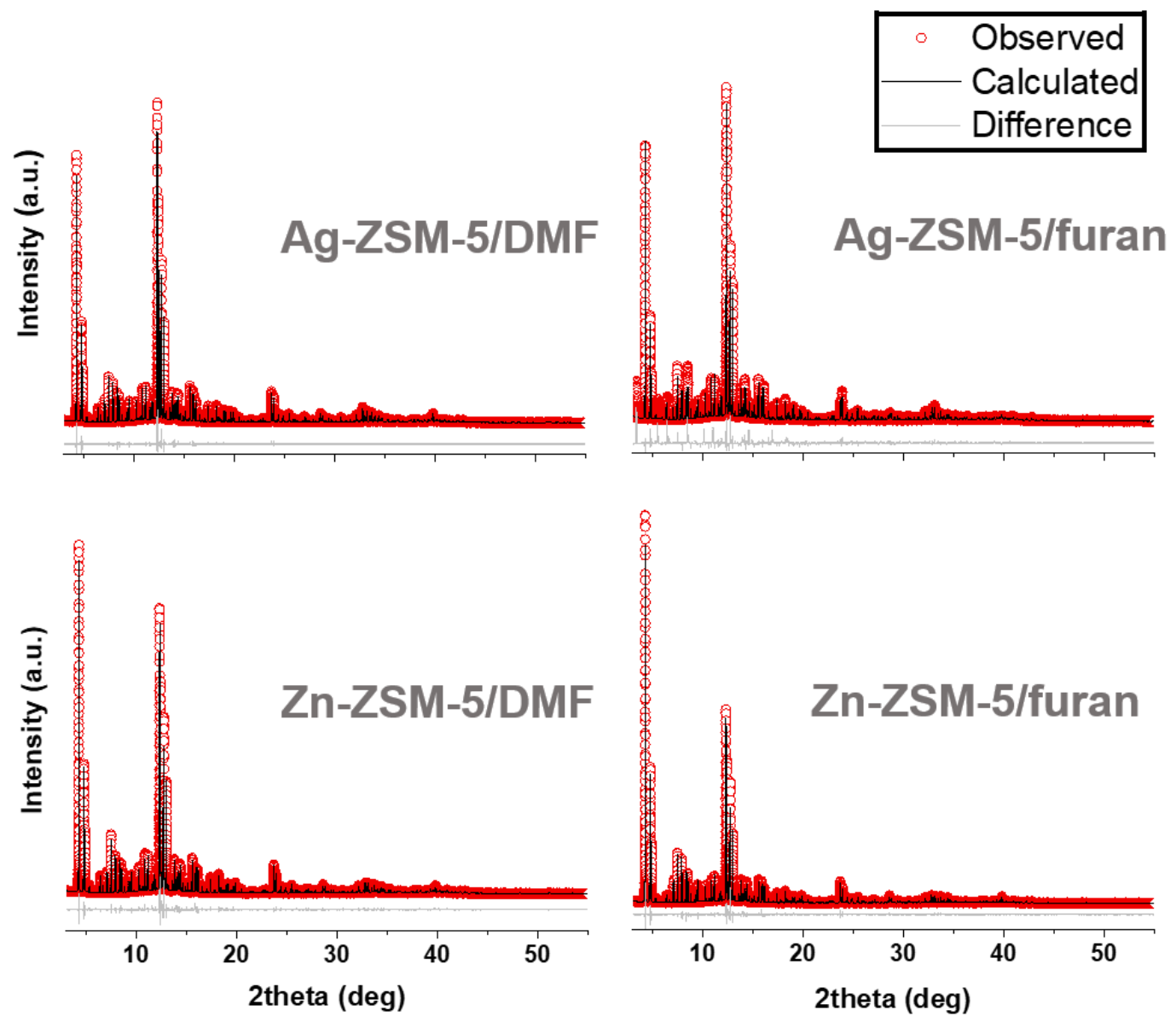

Figure 4. The SXRD patterns and Rietveld refinement profiles of Ag-ZSM-5/DMF, Ag-ZSM5/furan, Zn-ZSM-5/DMF, and Zn-ZSM-5/furan. Clear difference in the ratio Bragg's peaks can be seen, suggesting the ultra-high sensitivity of the synchrotron technique. 

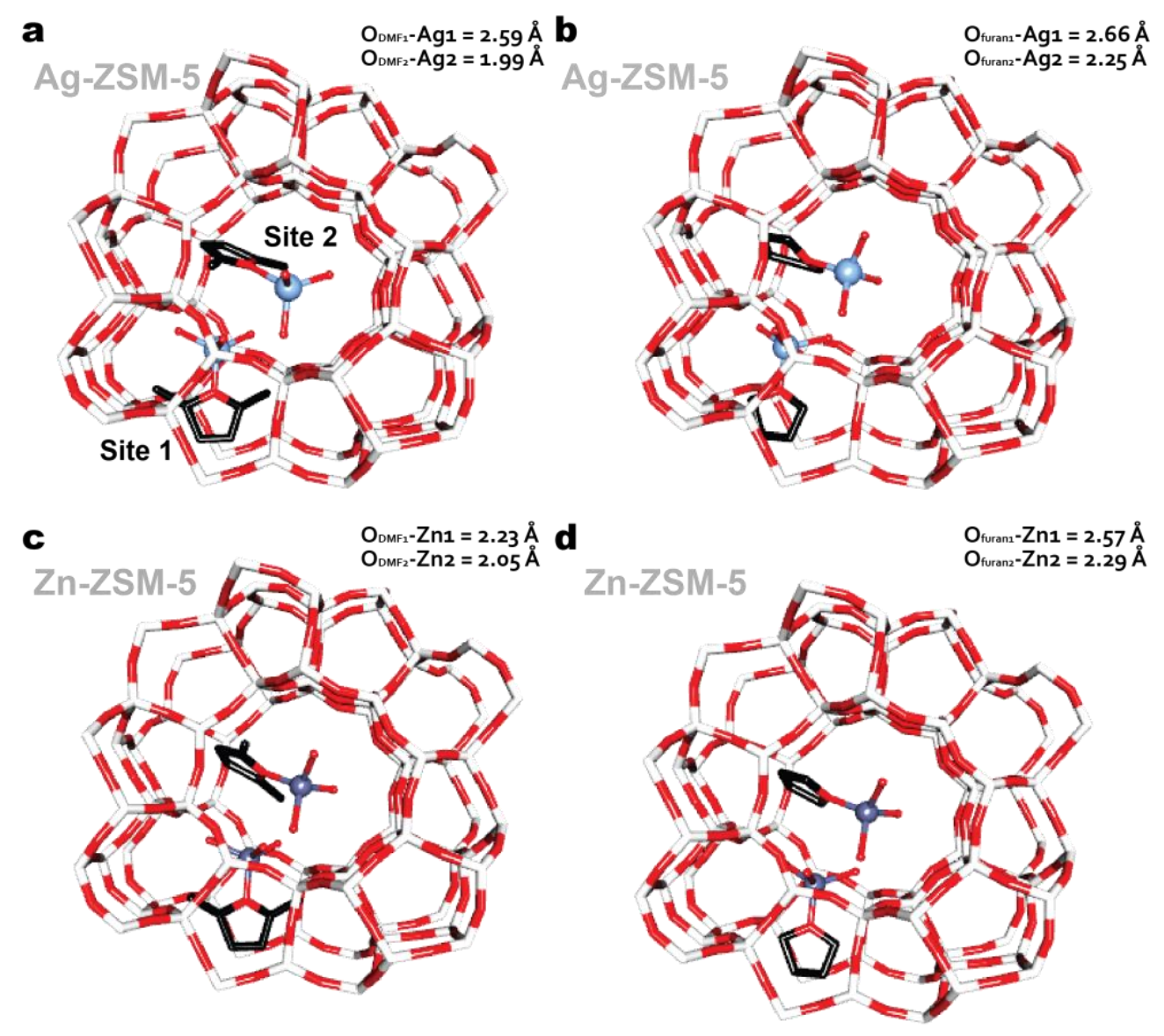

Figure 5. The refined crystal structures of (a) Ag-ZSM-5/DMF, (b) Ag-ZSM-5/furan, (c) Zn-ZSM5/DMF, and (d) Zn-ZSM-5/furan. The same colour scheme as applied in Figure 3, with light green $=\mathrm{Al}$, light blue $=\mathrm{Ag}$, green $=\mathrm{Cu}$, and purple $=\mathrm{Zn}$. See Table S5 for the detailed atomic parameters.

Even with the addition of metal LAS, it is found that the basicity of the furans also plays a critical role. Comparing $\mathrm{O}_{\mathrm{DMF} 1}-\mathrm{Zn} 1(2.23 \AA)$ with $\mathrm{O}_{\text {furan1 } 1-Z n 1}(2.57 \AA), \mathrm{O}_{\mathrm{DMF} 1}-\mathrm{Zn} 1$ is notably

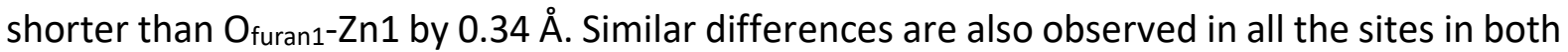
Ag- and Zn-ZSM-5.

Specifically, it should be noted the interatomic (or bond) distances in H-ZSM-5 and metal ionexchanged ZSM-5 could not be compared directly, as the inherent acidic properties between the BAS and metal LAS in zeolites are similar but exhibit some fundamental differences. $\mathrm{H}$ USY and H-ZSM-5 adsorb DMF (akin to furan) via the Al-O(H)-Si, by forming Al-OH( $\cdots$ DMF)-Si, thus $01-\mathrm{O}_{D M F}$ and $018-\mathrm{O}_{D M F}$ measure the interatomic distance between the $\mathrm{O}_{D M F}$ and the framework $01_{\mathrm{H}-\text { UsY }} / \mathrm{O} 18_{\mathrm{H}-\mathrm{ZSM}-5}$ atoms - via the bridging $\mathrm{H}$ atom. Whereas, $\mathrm{Ag}-\mathrm{ZSM}-5$ and $\mathrm{Zn}$ ZSM-5 adsorb DMF via Lewis acid-base interaction (by ligand exchange with water), where 
Ag2- $O_{D M F}$ and $Z n 2-O_{D M F}$ measure the 'real' interatomic distances between the $\mathrm{O}_{\mathrm{DMF}}$ atom and Lewis acidic metal moiety.

It is evident that the basicity-spatial arrangement relationship of the adsorbate species over the BAS and metal LAS in zeolites can be demonstrated in terms of thermogravimetric and structural evidence, which is consistent with the classical acid-base chemistry. Briefly, the more basic the probe adsorbate species is, the stronger acid-base adduct is formed giving shorter metal-adsorbate bond distance. It has been demonstrated the spatial properties of the adsorbate species can influence the catalytic performance [3]. 


\section{Conclusion}

In summary, by employing high-resolution SXRD with Rietveld refinement, we offer an extensive spatial understanding of the adsorbate interactions chemistry between probe molecules and acidic zeolites with and without doping of metal ions. By systematically studying DMF and furan as probe molecules, their adsorbate structures and interactions with the interior BAS and LAS of metal ion $\left(\mathrm{Ag}^{+}\right.$and $\left.\mathrm{Zn}^{2+}\right)$ in $\mathrm{H}-\mathrm{USY}$ and $\mathrm{H}-\mathrm{ZSM}-5$ zeolites have been determined. In essence, this descriptive study of surface acid-base chemistry on zeolites is not only merely a supplementary classification scheme on top of the traditional understanding but also provides a quantitative perspective to interrogate and optimise complex reactions catalysed by zeolites based on structural evidence. 


\section{References}

[1] J.W. Erisman, M.A. Sutton, J. Galloway, Z. Klimont, W. Winiwarter, Nat. Geosci. 1 (2008) 636-639.

[2] M.E. Davis, Microporous Mesoporous Mater. 21 (1998) 173-182.

[3] I.F. Teixeira, B.T.W. Lo, P. Kostetskyy, M. Stamatakis, L. Ye, C.C. Tang, G. Mpourmpakis, S.C.E. Tsang, Angew. Chem. Int. Ed. 55 (2016) 13061-13066.

[4] I.F. Teixeira, B.T.W. Lo, P. Kostetskyy, L. Ye, C.C. Tang, G. Mpourmpakis, S.C.E. Tsang, ACS Catal. (2018) 1843-1850.

[5] W.O. Haag, R.M. Lago, P.B. Weisz, Nature 309 (1984) 589-591.

[6] M. Boronat, A. Corma, ACS Catal. 9 (2019) 1539-1548.

[7] L. Ye, Q. Song, B.T.W. Lo, J. Zheng, D. Kong, C.A. Murray, C.C. Tang, S.C.E. Tsang, Angew. Chem. Int. Ed. 56 (2017) 10711-10716.

[8] Y.-T. Cheng, G.W. Huber, ACS Catal. 1 (2011) 611-628.

[9] Y.-T. Cheng, G.W. Huber, Green Chem. 14 (2012) 3114-3125.

[10] W. Lin, L. Ye, S. Wu, B. Lo, Y. Peng, P. Zhao, I. Mcpherson, J. Catal. (2018) 4214-4218.

[11] S. Yang, J. Sun, A.J. Ramirez-Cuesta, S.K. Callear, W.I.F. David, D.P. Anderson, R. Newby, A.J. Blake, J.E. Parker, C.C. Tang, M. Schröder, Nat. Chem. 4 (2012) 887-894.

[12] Q. Liu, A. Mace, Z. Bacsik, J. Sun, A. Laaksonen, N. Hedin, Chem. Commun. 46 (2010) 4502-4504.

[13] D.S. Wragg, M.G. O’Brien, F.L. Bleken, M. Di Michiel, U. Olsbye, H. Fjellvãg, Angew. Chem. Int. Ed. 51 (2012) 7956-7959.

[14] A.B. Pinar, L. Gómez-Hortigüela, L.B. McCusker, J. Pérez-Pariente, Chem. Mater. 25 (2013) 3654-3661.

[15] P. Zhao, L. Ye, Z. Sun, B.T.W. Lo, H. Woodcock, C. Huang, C. Tang, A.I. Kirkland, D. Mei, S. Chi, E. Tsang, J. Am. Chem. Soc. 140 (2018) 6661-6667.

[16] D.S. Wragg, D. Akporiaye, H. Fjellvåg, J. Catal. 279 (2011) 397-402. 
[17] B.T.W. Lo, L. Ye, J. Qu, J. Sun, J. Zheng, D. Kong, C.A. Murray, C.C. Tang, S.C.E. Tsang, Angew. Chem. Int. Ed. 55 (2016) 5981-5984.

[18] W. Dai, C. Wang, M. Dyballa, N. Guan, L. Li, Z. Xie, M. Hunger, W. Dai, C. Wang, M. Dyballa, G. Wu, N. Guan, L. Li, Z. Xie, M. Hunger, ACS Catal. (2015) 317-326.

[19] W.E. Farneth, R.J. Gorte, Chem. Rev. 95 (1995) 615-635.

[20] K. Pei, H. Li, J. Mol. Struct. 693 (2004) 141-144.

[21] P.A. Denis, Theor. Chem. Acc. 129 (2011) 219-227.

[22] E.L. First, C.E. Gounaris, J. Wei, C.A. Floudas, Phys. Chem. Chem. Phys. 13 (2011) 17339-17358.

[23] E.P.L. Hunter, S.G. Lias, J. Phys. Chem. Ref. Data 27 (1998) 413-656.

[24] S.P. Thompson, J.E. Parker, J. Potter, T.P. Hill, A. Birt, T.M. Cobb, F. Yuan, C.C. Tang, Rev. Sci. Instrum. 80 (2009).

[25] B.T.W. Lo, L. Ye, G.G.Z. Chang, K. Purchase, S. Day, C.C. Tang, D. Mei, S.C.E. Tsang, Appl. Catal. B Environ. 237 (2018) 245-250.

[26] B.T.W. Lo, L. Ye, C.A. Murray, C.C. Tang, D. Mei, S.C.E. Tsang, J. Catal. 365 (2018) 145152.

[27] S.M. Babitz, B.A. Williams, J.T. Miller, R.Q. Snurr, W.O. Haag, H.H. Kung, Appl. Catal. A Gen. 179 (1999) 71-86.

[28] A. Redondo, P.J. Hay, J. Phys. Chem. 97 (1993) 11754-11761.

[29] N. Katada, K. Suzuki, T. Noda, G. Sastre, M. Niwa, J. Phys. Chem. C 113 (2009) 1920819217.

[30] A. Nakamura, M. Narita, S. Narita, Y. Suzuki, T. Miyanaga, in:, J. Phys. Conf. Ser., IOP Publishing, 2014, p. 12033.

[31] E.S.C. Tsang, L. Ye, B. Lo, Q. Song, J. Zheng, D. Kong, C. Tang, Angew. Chem. Int. Ed. 56 (2017) 10711-10716.

[32] N. Nikbin, P.T. Do, S. Caratzoulas, R.F. Lobo, P.J. Dauenhauer, D.G. Vlachos, J. Catal. 
297 (2013) 35-43.

[33] R.F. See, R.A. Kruse, W.M. Strub, Inorg. Chem. 37 (1998) 5369-5375. 